\title{
ON THE EXISTENCE OF A SMALL CONNECTED OPEN SET WITH A CONNECTED BOUNDARY
}

\author{
BY F. BURTON JONES ${ }^{1}$ \\ Communicated by Edwin Moise, December 23, 1961
}

In a connected, locally connected, locally compact metric space with no local separating point it is rather easy to construct an arbitrarily small connected open set whose boundary is a subset of an arbitrary small continuum lying in its complement. In fact such sets form a topological basis for the space. However, it seems to be much more difficult to construct small connected open sets whose boundaries are connected. The author constructed such open sets (substituting something weaker for local compactness) [1, Theorem 33] in certain special plane-like spaces but efforts at that time to generalize the theorem failed. Now with the help of the partitioning technique (brick partitioning, in particular) the construction may be carried out successfully. ${ }^{2}$

Lemma. Suppose that (1) $U$ is a connected open proper subset of the connected, locally connected, compact metric space $M$ such that $\bar{U}=M$, (2) $p$ is a point of $U$ such that $M-p$ is connected and (3) no point of $M-p$ is a local separating point of $M$. Then if $\epsilon$ is a positive number, there exists a connected open point set $V$ such that (1) $p \in V \subset \bar{V} \subset U$, (2) $M-\bar{V}$ is connected and (3) if $x \in M-V, d(x, V)<\epsilon$.

INDICATION OF PROOF. Let $F$ denote the boundary of $U$. Without loss of generality we shall assume that $3 \epsilon$ is less than $d(p, F)$. Being compact and locally connected, $M$ has property $S$. By Theorem 8 of [2] there exists a sequence $G_{1}, G_{2}, \ldots$ such that $G_{i}$ is a brick $(1 / i)$ partitioning of $M$ and $G_{i+1}$ is a refinement of $G_{i}$. Let $B_{i}$ denote the subcollection of elements $g$ of $G_{i}$ such that $\bar{g} \cdot F \neq 0$ and let $H_{i}$ denote the subcollection of $G_{i}$ consisting of the elements of $B_{i}$ together with all other elements of $G_{i}$ which are separated from $p$ by $\bar{B}_{i}^{*}$.

There exists a value of $i$ such that each point of $\bar{H}_{i}^{*}$ is within $\epsilon / 4$ of $F$. For suppose on the contrary that for each $i, \bar{H}_{i}^{*}$ contains a point $q_{i}$ such that $d\left(q_{i}, F\right) \geqq \epsilon / 4$. Let us suppose that $\left\{q_{i}\right\}$ converges to $q$ (for certainly some subsequence converges). Since $d(q, F) \geqq \epsilon / 4, q$ belongs to $U$ and there is an arc $p q$ from $p$ to $q$ lying in $U$. Now let $i(q)$ be a value of $i$ large enough so that if $g_{1}, g_{2} \in G_{i}, \bar{g}_{1} \cdot p q \neq 0$ and $\bar{g}_{2} \cdot F \neq 0$,

${ }^{1}$ Work done under NSF G-9418.

${ }^{2}$ I wish to thank Professor A. H. Stone for calling my attention to the fact that the problem was still unsolved. 
then $\bar{g}_{1} \cdot \bar{g}_{2}=0$. Obviously the interior of the sum of the closures of elements of $G_{i(q)}$ whose closures intersect $p q$ does not intersect $\bar{H}_{i}^{*}$ for $i \geqq i(q)$. But since this interior is a connected open set containing $p+q$, it contains $q_{i}$ for infinitely many values of $i$. This is a contradiction.

Furthermore, letting $K_{i}$ denote $G_{i}-H_{i}$, there exists a value of $i$ such that every point of $F$ is within $\epsilon / 4$ of int $\left(\bar{K}_{i}^{*}\right)$. Let $i_{1}$ be the larger of these two values of $i$ and let $V_{1}$ denote $\operatorname{int}\left(\bar{K}_{i_{1}}^{*}\right)$.

Clearly $V_{1}$ is a connected open subset of $U$ containing $p$ and $M_{1}=\bar{V}_{1}$ is a subcontinuum of $U$ having property $S$. Let $C_{1}, C_{2}, \cdots, C_{n_{1}}$ denote the components of $M-M_{1}$. Since the partitioning is a brick partitioning, these components cover $F$ and their closures are nonintersecting. Since $p$ does not separate $M$, there exists an arc $a b$ in $M-p$ irreducible from $\bar{C}_{1}$ to $\bar{C}_{2}+\bar{C}_{3}+\cdots+\bar{C}_{n_{1}}$. Let $Q$ denote the component of $V_{1}-p$ containing $a b-(a+b) ; \bar{Q}$ has property $S$. Since $p$ does not separate $\bar{Q}$ and no point of $\bar{Q}-p$ is a local separating point of $\bar{Q}$, no pair of points of $\bar{Q}$ separates $\bar{Q}$. By Theorem 17 of [2] there exists an arc $T^{\prime}$ from $a$ to $b$ lying in $\bar{Q}-p$ which does not separate $\bar{Q}$. Let $T_{1}$ denote a subarc of $T^{\prime}$ irreducible from $\bar{C}_{1}$ to $\bar{C}_{2}+\bar{C}_{3}+\cdots+\bar{C}_{n_{1}}$.

The arc $T_{1}$ does not separate $\bar{Q}$ and lies (except for its endpoints) in $Q$. In fact $p+\left(Q-T_{1}\right)$ is connected. To see that this is true, let $x$ denote a point of $Q-T^{\prime}$. In $\bar{Q}-T^{\prime}$ there exists an arc $x p$. There exists an integer $j>i_{1}$ such that (1) if $g_{1}, g_{2} \in G_{j}, \bar{g}_{1} \cdot x p \neq 0$ and $\bar{g}_{2} \cdot T^{\prime} \neq 0$, then $\bar{g}_{1} \cdot \bar{g}_{2}=0$, and (2) if $g_{1}, g_{2} \in G_{j}, \bar{g}_{1} \cdot(x+p) \neq 0$ and $\bar{g}_{2} \cdot\left(M-V_{1}\right) \neq 0$, then $\bar{g}_{1} \cdot \bar{g}_{2}=0$. The interior $I$ of the sum of the closures of the elements of $G_{j}$ lying in $V_{1}$ whose closures intersect $x p$ is a connected open subset of $V_{1}$ containing no point of $T^{\prime}$ and no point of the boundary of $V_{1}$. But $I$ contains $p+x$. In $I$ there is an arc $p x$. But $p x$ is obviously a subset of $p+\left(Q-T^{\prime}\right)$. So $p+\left(Q-T^{\prime}\right)$ is connected. Since no point of $M_{1}-p$ is a local separating point of $M_{1}$, every point of an arc in $M_{1}$ is a boundary point of the arc relative to $M_{1}$. Hence $p+\left(Q-T_{1}\right)$ is connected.

Clearly $U_{1}=V_{1}-T_{1}$ is connected and $M_{1}=\bar{U}_{1}$. Let $F_{1}$ be the boundary of $U_{1}$.

Now the entire process may be repeated using $U_{1}, M_{1}$, and $F_{1}$ for $U, M$, and $F$ respectively but being sure that $i_{2}$ is a value of $i$ such that each point of $\bar{H}_{i}^{*}$ is within $\epsilon / 4 n_{1}$ of $F_{1}\left(n_{1}\right.$ was the number of the components $C_{i}$ ). The fact that $p$ does not separate $M$ need not be inherited by $M_{1}$. By taking $T_{2}$ to join the boundaries of different components of $M-M_{2}$, the number of these components is again reduced by at least one. Hence in $n_{1}$ (or less) steps $V_{n_{1}}$ is the $V$ called for in the lemma. 
TheOREM. Suppose that $S$ is a connected, locally connected, locally compact metric space which contains no local separating point. Then if $p$ is a point of an open subset $R$ of $S$, there exists a connected open set $D$ such that $p \in D \subset R$ and the boundary of $D$ is connected.

Proof. By Theorem 2.4 of [3] there exists a connected, uniformly locally connected open proper subset $U$ of $R$ such that $U$ contains $p$ and $\bar{U}$ is compact. Let $F$ denote the boundary of $U$ and $M=U+F$. Hence by the lemma there exists a connected open set $V_{1}$ such that (1) $p \in V_{1} \subset \bar{V}_{1} \subset U$, (2) $M-\bar{V}_{1}$ is a connected open (rel $M$ ) subset of $M$ containing $F$ and (3) if $x$ is a point of $M-V_{1}$ then $d\left(x, V_{1}\right)<1$. Consider the decomposition space $M_{1}$ of $M$ in which the only nondegenerate element $P_{1}$ is $\bar{V}_{1}$. Now $P_{1}$ does not separate $M_{1}$ and no other point of $M_{1}$ is a local separating point of $M_{1}$ relative to $M_{1}$. We can now reapply the lemma to get $V_{2}$ with the properties of $V$ of the lemma so that if $x$ is a point of $M_{1}-V_{2}\left(=M-V_{2}^{*}\right)$, then $d\left(x, V_{2}^{*}\right)$ $<1 / 2$ (here $V_{2}^{*}$ is a subset of $S$ and $d$ is the distance function for $S$ ). This process may be continued so that $p \in V_{1} \subset \bar{V}_{1} \subset V_{2}^{*} \subset \bar{V}_{2}^{*} \subset \ldots$. where if $x$ is a point of $M-V_{n}^{*}$, then $d\left(x, V_{n}^{*}\right)<1 / n$. Since $M-\bar{V}_{n}^{*}$ is connected, $\Pi\left(M-V_{n}^{*}\right)$ is a continuum containing $F$. Furthermore it is the boundary of $\Sigma V_{n}^{*}$. So $\Sigma V_{n}^{*}$ is the required connected open subset $D$ of $R$ containing $p$.

\section{BIBLIOGRAPHY}

1. F. Burton Jones, Certain equivalences and subsets of a plane, Duke Math. J. 5 (1939), 133-145.

2. R. H. Bing, Complementary domains of continuous curves, Fund. Math. 36 (1949), 303-318.

3. R. L. Wilder, Topology of manifolds, Amer. Math. Soc. Colloq. Publ. Vol. 32, Amer. Math. Soc., 1949.

University of North Carolina 